\title{
Carrier Lifetime Assessment in Integrated Ge Waveguide Devices
}

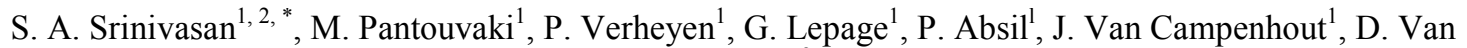 \\ Thourhout $^{2}$ \\ ${ }^{1}$ Imec, Kapeldreef 75, Heverlee B-3001, Belgium \\ ${ }^{2}$ Photonics Research Group, Dept. of Information Technology, Ghent University - imec \& Center for Nano- and Biophotonics, Ghent \\ University, St. Pietersnieuwstraat 41, 9000 Ghent, Belgium \\ "Authore-mail address: ashwyn.srinivasan@imec.be
}

\begin{abstract}
Carrier lifetimes in Ge waveguides on Si are deduced from time-resolved pump-probe spectroscopy. For a 1 $\mu \mathrm{m}$ wide Ge waveguide, a lifetime of $1.6 \mathrm{~ns}$ is estimated for a carrier density of around $2 \times 10^{19} \mathrm{~cm}^{-3}$.
\end{abstract}

Index Terms - Waveguide modulator; Optical interconnects; Silicon photonics

\section{INTRODUCTION}

Qilicon photonics is widely considered as a potential Solution in solving the bandwidth-power density bottleneck of electrical interconnects for future CMOS systems [1]. Ge waveguides (WG) with an embedded p-i-n diode integrated on a silicon photonics platform (Fig. 1) can be used as a building block for a number of devices such as Ge photodetectors [2], Ge electro-absorption modulators [3], as well as Ge variable optical attenuators (VOA) and Ge lasers (with high n-type doping and tensile strained active region [4]). In this work, we extract carrier lifetimes in Ge waveguides grown on $\mathrm{Si}$, using time-resolved infrared transmission pump-probe spectroscopy. This experimental method and analysis enable benchmarking and subsequent optimization of carrier-injection based devices, such as VOAs and lasers. In this work, we study the impact of surface recombination on carrier lifetime in sub-micron Ge WGs.

\section{II.DESIGN AND FABRICATION OF GE P-I-N WG}

A set of Ge WGs with embedded p-i-n diode having a length of $8 \mu \mathrm{m}$ and varying width $(400 \mathrm{~nm}, 600 \mathrm{~nm}, 800 \mathrm{~nm}$ and $1 \mu \mathrm{m})$ are studied here. They were fabricated in imec's $200 \mathrm{~mm} \mathrm{Si}$ photonics platform [5]. The $\mathrm{Ge} \mathrm{WG}$ is adiabatically coupled to an SOI WG using poly-Si tapers. TE mode grating couplers with a peak wavelength of $1595 \mathrm{~nm}$ were used for coupling light to and from the chip. The p-i-n Ge WGs are $350 \mathrm{~nm}$ thick and grown on top of the recessed Si layer of the SOI wafer as shown in Fig. 1. Such a device was operated in reverse bias as an electro-absorption modulator in [3] but can in forward bias also be used as a VOA or Ge laser, provided with appropriate strain and high n-type doping [4].

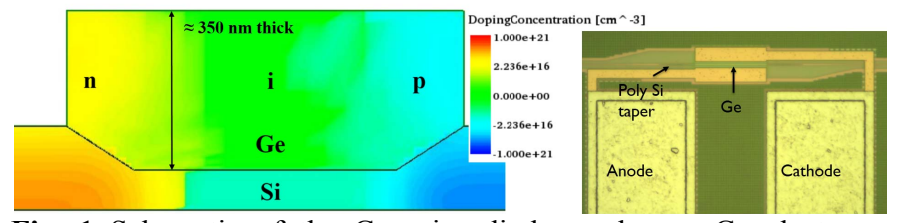

Fig. 1 Schematic of the Ge p-i-n diode used as a Ge electroabsorption modulator ( $1 \mu \mathrm{m}$ wide) and a microscope image of the fabricated Ge p-i-n WG [3].

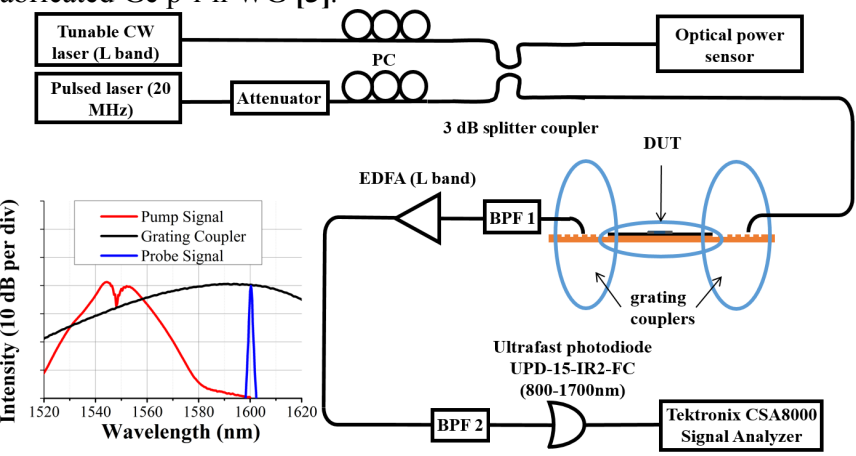

Fig. 2 Measurement schematic with band pass filters (BPF1 and BPF2) centered at probe wavelength to suppress the pump and noise from EDFA with respect to the probe signal. The spectrum of probe signal power, pump signal power and grating coupler efficiency are shown in the inset.

\section{EXPERIMENT}

We used a mode-locked femtosecond fiber laser from Calmer laser as the pump and a CW tunable laser source as the probe [6]. The pump signal has a center wavelength of $1548.57 \mathrm{~nm}$ with a pulse width of $0.235 \mathrm{ps}$ while the probe signal has a center wavelength of $1600 \mathrm{~nm}$. The light is coupled by the grating couplers into the TE-like waveguide mode and the absorption is dominated by the $\Gamma$-hh band gap $(\sim 1580 \mathrm{~nm})$ [3]. The probe signal is set at $1600 \mathrm{~nm}$ so that it can be filtered out easily from the pump and is below the $\Gamma$-hh band-gap of Ge. The pump is operated at a repetition rate of $20 \mathrm{MHz}$ with an average output power of around $7.9 \mathrm{~mW}$ and peak energy of 400 pJ. A $10 \mathrm{~dB}$ attenuator is placed after the pump source to avoid any self-phase modulation in the fiber as well as two-photon absorption in the SOI WG. Time-resolved transmission data is extracted from a Tektronix Signal Analyzer, which is triggered by the pulsed laser. The time resolution of the measurement is limited by the 15 ps rise time of the photodiode. 


\section{DISCUSSION AND RESULTS}

When pumping the device under test, the pump signal will be absorbed creating a high density of electrons and holes. These carriers will decay in time due to recombination. The dynamics of these carriers are probed using a $\mathrm{CW}$ signal at $1600 \mathrm{~nm}$ which suffers from the pump-induced excess free carrier absorption (FCA) and optical bleaching effect (OBE). The power of the probe signal is monitored using a high-speed oscilloscope as shown in the inset of Fig. 3. From the excess loss, and using the equations for FCA and OBE from [4], we can estimate the average carrier concentration in the Ge WG as function of time (Fig. 3) and the carrier lifetimes at these carrier densities. The latter are shown in Fig. 4, for a carrier concentration of approximately $2 \times 10^{19} \mathrm{~cm}^{-3}$, and for different Ge WG widths.

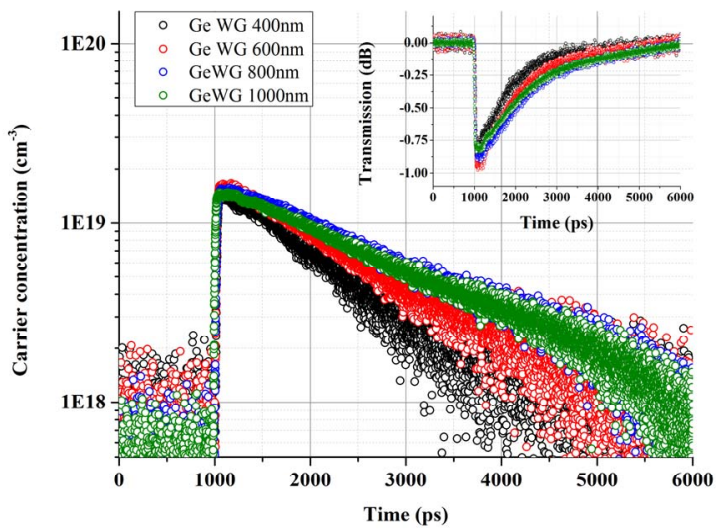

Fig. 3 Time resolved extracted carrier concentration in Ge WG from transmission measurements in $8 \mu \mathrm{m}$ long device (inset) for different width.

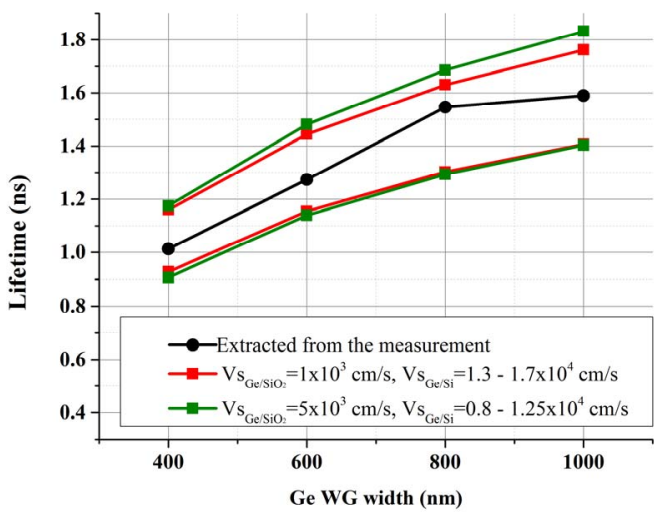

Fig. 4 Extracted carrier lifetimes for different Ge WG widths. The colored data points are the modeled carrier lifetimes assuming a fixed surface recombination velocity $\left(\mathrm{V}_{\mathrm{s}}\right)$ for $\mathrm{Ge} / \mathrm{SiO}_{2}$ and $\mathrm{Ge} / \mathrm{Si}$. The data above the measurement correspond to under-estimation of $\mathrm{V}_{\mathrm{s}}$ and those below the measurement correspond to overestimation of $\mathrm{V}_{\mathrm{s}}$ for $\mathrm{Ge} / \mathrm{Si}$ interface for a fixed $\mathrm{V}_{\mathrm{s}}$ for $\mathrm{Ge} / \mathrm{SiO}_{2}$ interface.

For such a carrier concentration, Auger recombination and radiative recombination from the direct and indirect band gap are expected to be small as compared to defect assisted SRH recombination and surface recombination at $\mathrm{Ge} / \mathrm{SiO}_{2}$ and $\mathrm{Ge} / \mathrm{Si}$ interfaces [8]. In this paper, we assume that the carriers generated by the pump are distributed uniformly across the length of the WG and that carrier lifetimes are not affected by diffusion effects. The dependence of the carrier lifetime on the WG width suggests that the $\mathrm{Ge} / \mathrm{SiO}_{2}$ and $\mathrm{Ge} / \mathrm{Si}$ interfaces in the side walls have a significant impact on the recombination rate. This impact is reduced as we increase the volume/surface-area ratio when using wider devices. Assuming that the lifetime is surface recombination limited, we extract the surface recombination velocity for each interface as shown in Fig. 4 using the related equation in [8]. In this extraction, we assume the top and side interfaces between $\mathrm{Ge}$ and $\mathrm{SiO}_{2}$ and the bottom and sloped interfaces between $\mathrm{Ge}$ and $\mathrm{Si}$ to behave similarly and fit the recombination velocities (Fig. 4). From this fitting, we estimate the extracted recombination velocity for the $\mathrm{Ge} / \mathrm{Si}$ interface to be less than $2 \times 10^{4} \mathrm{~cm} / \mathrm{s}$ and the recombination velocity for the $\mathrm{Ge} / \mathrm{SiO}_{2}$ to be the range $1-5 \times 10^{3} \mathrm{~cm} / \mathrm{s}$. The recombination velocities for $\mathrm{Ge} / \mathrm{Si}$ interface are in good agreement with those reported in [7,9]. The contribution to carrier recombination at $\mathrm{Ge} / \mathrm{SiO}_{2}$ sidewall layers can be suppressed in future devices by using better passivation coatings.

\section{CONCLUSION}

We investigated carrier lifetimes of Ge p-i-n WG on Si with different widths using time-resolved infrared transmission pump-probe spectroscopy. The lifetime of free electrons and holes increases for wider devices, thus revealing the impact of side walls of the WG. For a $1 \mu \mathrm{m}$ wide WG, we estimate the lifetime to be $1.6 \mathrm{~ns}$. We also estimate the $\mathrm{Ge} / \mathrm{Si}$ interface to have a recombination velocity of $<2 \times 10^{4} \mathrm{~cm} / \mathrm{s}$.

\section{ACKNOWLEDGEMENTS}

This work was supported by imec's industry-affiliation program on Optical I/O.

\section{REFERENCES}

[1] D.A.B. Miller, et al. "Device Requirements for Optical Interconnects to Silicon Chips", Proc. IEEE vol. 97, no. 7, pp. 1166-1185, Jul. 2009.

[2] H. Chen, et al. "High responsivity low-voltage $28 \mathrm{~Gb} / \mathrm{s}$ Ge p-i-n photodetector with silicon contacts", Journal of Light. Tech., 33(4), p.820-824, 2015.

[3] S. Gupta, et al. " $50 \mathrm{GHz}$ Ge Waveguide Electro-Absorption Modulator Integrated in a 220nm SOI Photonics Platform", Optical Fiber Communication Conference, OSA Technical Digest, 2015, paper Tu2A.4.

[4] J. Liu, et al. 'Tensile strained, n-type Ge as a gain medium for monolithic laser integration on Si", Optics Letters, 15(19), 1127277, 2007.

[5] P. Verheyen, et al. "Highly Uniform $25 \mathrm{~Gb} / \mathrm{s}$ Si Photonics Platform for High-Density, Low-Power WDM Optical Interconnects", in Advanced Photonics for Communications, OSA Technical Digest, 2014, paper IW3A.4.

[6] R. Van Laer, et al. "Interaction between light and highly confined hypersound in a Silicon photonics nanowire", Nature Photonics, 9, 199-203, 2015.

[7] R. Geiger, et al. "Excess carrier lifetimes in Ge layers on Si", Applied Physics Letters, 104(6), 062106, 2014.

[8] L. A. Corden, et al. Diode Lasers and Photonic Integrated Circuits, (Wiley, New Jersey, 2012), Chapter 4.

[9] M. Virgilio et al. "Photoluminescence, recombination rate, and gain spectra in optically excited n-type and tensile strained germanium layers", Journal of Applied Physics, 114(24), 243102, 2013. 